\title{
Formação em saúde e produção de vínculo: uma experiência PET-Saúde na rede de Niterói, RJ, Brasil
}

Carla de Gouvêa dos Santos ${ }^{(a)}$

Fernanda Tosta de Alcântara Portugal(b) Maria Alice Bastos Silva ${ }^{(c)}$

Ândrea Cardoso de Souza ${ }^{(\mathrm{d})}$ Ana Lúcia Abrahão(e)

\section{Introdução}

A formação profissional no campo da saúde tem sido muito discutida nos últimos anos, com movimento claro de buscar articular a diversidade nos processos formativos com o mundo do trabalho, voltando atenção aos novos modos de ensinar a ser profissional.

Tem-se, no sistema educacional brasileiro, uma variedade de desenhos curriculares e práticas pedagógicas que se mantêm desarticulados dos cenários de prática e desconectados de outras disciplinas. A orientação dada à formação do profissional de saúde retrata a predominância dos currículos de conteúdos sequenciais e desconsideram que alunos, professores e profissionais percorram, com singularidade, trajetórias educacionais e experienciais, traçando seu próprio projeto acadêmico e profissional ${ }^{1}$

O Programa de Educação pelo Trabalho para Saúde (PET-Saúde) está estruturado sobre a tentativa de elaborar outros modos de ensinar em saúde que contemplem as vivências dos alunos e adotem a perspectiva da interdisciplinaridade, proporcionando a entrada dos alunos cada vez mais cedo aos cenários de prática.

Os estágios e vivências proporcionados aos estudantes pelo PET-Saúde objetivam adequar a formação às necessidades do Sistema Único de Saúde (SUS). No rol dos objetivos do PET, constam: desenvolver atividades acadêmicas em padrões de qualidade de excelência, mediante grupos de aprendizagem tutorial de natureza coletiva e interdisciplinar ${ }^{2}$.

A Universidade Federal Fluminense (UFF), em parceria com a Fundação Municipal de Saúde de Niterói (FMS), participa da proposta desde 2008, contemplando alunos dos cursos de Medicina, Enfermagem, Nutrição, Odontologia, Farmácia, Educação Física e Psicologia. No município, os grupos PET são organizados a partir de redes de cuidados. Em 2012, um desenho considerou o diagnóstico da situação de saúde da cidade e os principais problemas a serem enfrentados nos territórios para atender as necessidades de saúde da população.

Nesse formato de condução da formação, alunos dos diferentes cursos atuam, conjuntamente, sobre os mais variados problemas, interagindo diretamente com

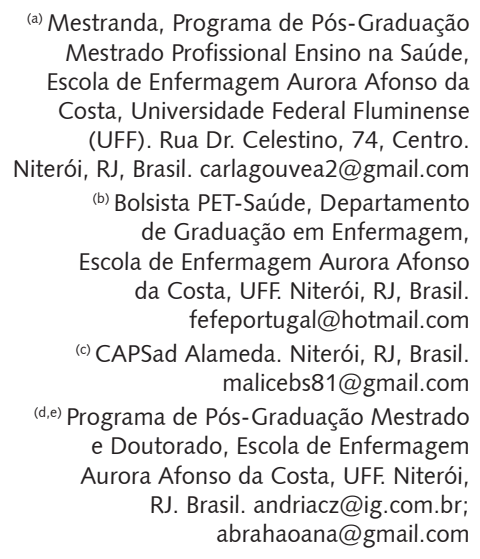

(a) Mestranda, Programa de Pós-Graduação Mestrado Profissional Ensino na Saúde, Escola de Enfermagem Aurora Afonso da Costa, Universidade Federal Fluminense (UFF). Rua Dr. Celestino, 74, Centro. Niterói, RJ, Brasil. carlagouvea2@gmail.com (b) Bolsista PET-Saúde, Departamento de Graduação em Enfermagem, Escola de Enfermagem Aurora Afonso da Costa, UFF. Niterói, RJ, Brasil. fefeportugal@hotmail.com

(c) CAPSad Alameda. Niterói, RJ, Brasil. malicebs81@gmail.com

(d,e) Programa de Pós-Graduação Mestrado e Doutorado, Escola de Enfermagem Aurora Afonso da Costa, UFF. Niterói, RJ.Brasil.andriacz@ig.com.br; abrahaoana@gmail.com 
os profissionais do serviço e o preceptor que os acompanha, independente do vínculo profissional. Ou seja, o aluno de Farmácia pode ter, como preceptor, um profissional de Odontologia e ambos atuarem com profissionais de Enfermagem, por exemplo. Dessa forma, o PET busca a formação interdisciplinar, ao mesmo tempo em que coloca um embaraço para o professor e profissionais do serviço, ao serem desafiados pela interprofissionalidade, à medida que são convocados a trabalhar com alunos de diferentes núcleos de conhecimento.

Este estudo tem por objetivo relatar a experiência de uma dupla aluno/preceptor no desafio de ampliar o debate sobre a potência de provocar espaço de produção de vínculo no ambiente de trabalho, articulado à formação em Saúde.

\section{Desenhando nova cartografia}

Utilizou-se o recurso metodológico da narrativa para relatar experiências vivenciadas por uma acadêmica de Enfermagem, bolsista do Programa de Educação pelo Trabalho para Saúde, e sua preceptora, uma assistente social. As narrativas não são apenas o produto de experiências individuais, pois foram construídas dialogicamente, utilizando-se de um contexto cultural que compartilha informações. Há três tipos de narrativas: as breves, as de vivências e as narrativas populares ${ }^{3}$. Neste estudo temos uma narrativa de vivência: acompanhamento do percurso de um usuário na rede de atenção psicossocial, especificamente em um serviço de álcool, crack e outras drogas (CAPS/ad), pelos diversos cenários da rede de atenção em saúde no município de Niterói. As narrativas de vivências são mais amplas, incluindo na história da vivência de uma pessoa: sensações, percepções, interpretações e indagações.

Para apresentação desta narrativa, aluna e preceptora pactuaram previamente a seleção dos trechos e detalhes que poderiam ou não ser ditos, e de que forma apareceriam no relato. Logo, optou-se por nomear os sujeitos com codinomes. Os autores reiteram que, ao selecionar detalhes para compor sua história, a pessoa está deixando em evidência o que considera importante para o relato. No caso deste estudo, obteve-se o conteúdo que compõe a narrativa por meio da observação participante no acompanhamento do usuário pela rede de saúde. Os percursos vividos foram registrados em diários de campo constituídos durante o desenvolvimento das atividades no CAPS/ad e nos circuitos percorridos por elas (aluna/preceptora) no período de outubro de 2013 a maio de 2014. Como ferramenta de pesquisa, empregou-se o método do aluno-sombra.

O método sombra é pautado no princípio do acolhimento e fortalecimento de vínculo ${ }^{4}$. Neste método, utilizado com a orientação da coordenação do PET-Saúde/UFF, acredita-se que a atuação em redes de atenção pressupõe arranjos pedagógicos em diferentes níveis de assistência, de modo a favorecer uma ampliação do cenário de prática desenhado. O aluno se coloca ao lado do usuário e segue seus passos por todos os lugares que o usuário se locomove dentro do serviço, andando junto dele, fazendo sombra na consulta, no exame, na sala de espera. O método tem a finalidade de mapear a rede de saúde por meio dos percursos e apontamentos do usuário: o que ele diz ser fácil ou difícil ao acessar os serviços, e não o que ou como os profissionais disponibilizam os serviços como sendo o melhor fluxo.

Acontece que, como acompanhamos de pertinho esses usuários, acabamos por estreitar e ampliar redes de relações: com o usuário, os familiares, a equipe do serviço e profissionais de outros serviços. Isso permitiu enxergar pontos obscuros que dificultam a assistência; por outro lado, essa rede de relações do sombra possibilita a criação de vínculos de confiança e segurança, que podem dar permissão/autorizar ou não a produção do cuidado integral.

Os diários da preceptora e da aluna foram transcritos no formato de escrita livre, posteriormente relidos e debatidos, suscitando reflexões de natureza prática, que deram margem à elaboração do eixo norteador "Desafios e contribuições das estratégias pedagógicas empregadas no PET-Saúde para formação em serviço na graduação em saúde". Este se relacionou ao objetivo do estudo, mas, sobretudo, para que, em torno dele, girasse a apresentação desta experiência, de modo que relações estabelecidas em momentos distintos, vivenciados em dupla e individualmente, pudessem evidenciar aspectos da produção de vínculo articulado à formação em serviço pelo PET-Saúde. 


\section{O vivido: interfaces, tecendo conexões possíveis}

O Centro de Atenção Psicossocial (CAPS) é um dispositivo importante na construção de redes de serviços assistenciais. Este serviço é também um dos cenários de prática para o grupo de aprendizagem do PET-Saúde da Universidade Federal Fluminense. Um serviço de saúde mental territorializado, especializado na assistência a pessoas que fazem uso abusivo de álcool e outras drogas, o CAPS/ad oferece, diariamente, acolhimento multiprofissional à demanda referenciada e espontânea.

A chegada ao local definido para desenvolvimento das atividades do PET-Saúde é um momento de expectativas para todos: profissionais do serviço, os usuários, estudantes e professores. Ainda não nos conhecemos e temos pela frente um mundo desconhecido e novo. O trabalho e a formação em saúde se desenrolam num emaranhado de processos que requer muito dos nossos sentidos, para que possamos compreender a dimensão das necessidades de saúde que atingem a vida das pessoas ou coletividade. Nosso grupo tutorial esteve composto por 12 acadêmicos, oriundos dos cursos de: Enfermagem, Medicina, Odontologia, Educação Física, Farmácia e Psicologia. Contou com seis professores-tutores e três preceptores do CAPS/ad. Eles acompanhavam uma dupla de alunos que eram de cursos diferentes. Os primeiros dias do grupo são reservados a observar o funcionamento do serviço, conhecer o processo de trabalho da equipe, a dinâmica de circulação dos usuários.

Aos poucos, nos aproximamos de outros cenários, é quando começamos a identificar a vida local. As atividades de formação em serviço são elaboradas e discutidas em conjunto - tutor, preceptor e aluno, incluindo outros profissionais do CAPS. Toda equipe se reúne uma vez por semana para discussão sobre o cotidiano do serviço e os projetos terapêuticos dos usuários que estão em diferentes momentos no tratamento. Esse é um dos momentos de revisão, avaliação e colaboração entre profissionais em formação, professores e profissionais do serviço. Pode-se contar que, nesses encontros, há momentos de muitas interrogações, sobretudo de rever as estratégias terapêuticas traçadas. É nesse momento que o aluno exercita o trabalho interdisciplinar.

\section{O andar do usuário na rede municipal de saúde}

Tivemos, como uma das atividades de formação em serviço no PET, o acompanhamento de um usuário por meio do aluno-sombra, um modo diferente de investigar e aprender em saúde. O aluno elege um usuário considerando questões pertinentes ao processo saúde-doença, a partir daí, segue os passos desse usuário no intento de verificar dificuldades e facilidades ao acessar serviços e ações de saúde. O aluno na função de sombra também tem por objetivo realizar o diagnóstico da rede levando em conta a fala do usuário, que nos aponta para o fato de como a rede garante acesso. Outra função importante foi participar na construção do Projeto Terapêutico Singular (PTS). Este possibilita a participação do usuário e, consequentemente, a construção de sua autonomia, considerando as necessidades individuais e o histórico do usuário que está inserido em um determinado contexto. Pode ser estabelecido por meio da atuação do profissional que é a referência ao usuário-família, ou do profissional com todos os membros da equipe, por meio de discussões referentes à necessidade terapêutica individual de cada caso ${ }^{5}$.

O caso eleito para esta narrativa é o do 'Senhor G'. Usuário de álcool com vários episódios de internação em hospitais psiquiátricos, transtorno mental, com deficiência de autocuidado, risco elevado de complicações cardiovasculares por controle inadequado da hipertensão, diabetes, e frequência irregular no CAPS/ad. O plano inicialmente traçado para o 'Senhor $G^{\prime}$, pela equipe multiprofissional, a qual passamos a integrar, consistiu em algumas etapas: elaboração de diagnóstico, responsabilidade de cada sujeito envolvido com o projeto (incluindo usuário-família-equipe), e tempo previsto para alcance de cada objetivo traçado.

Assim, o PTS do 'Senhor G' incluía: visitas no âmbito familiar e ambiente de convivência social, levantamento de hábitos culturais, alimentares, condições econômicas e participação nas atividades intra e extra CAPS. Foi proposto comparecimento três vezes na semana à unidade; as atividades sempre escolhidas e decididas por ele. Nosso papel era ofertar ações mostrando benefícios e estimular que ele próprio apontasse as atividades de seu interesse. Contudo, o PTS do 'Senhor G' precisou 
de muitos ajustes, extrapolou o tempo previsto e não atingiu as ações programadas. Do itinerário percorrido com o 'Senhor G', podemos destacar alguns pontos críticos, o principal deles diz respeito à organização da rede assistencial. Existe fragilidade na comunicação entre os setores e serviços; os registros de prontuário fornecem pouca informação.

$\mathrm{Na}$ última internação, o 'Senhor G' recebeu alta hospitalar e aguardou, por duas semanas, sem que fosse mobilizado qualquer meio de retorno para casa. As proposições do PTS alcançaram questões focais e laterais na rede social dele, entretanto a sustentabilidade do projeto na produção de autonomia necessita de articulação efetiva, visto o que constatamos em nossas andanças: mesmo com os benefícios que conquistou no início do tratamento, como o "De Volta para Casa", atualmente este recurso não mais possibilita continuar morando com a irmã com qualidade de vida e prescindir das internações. Ele tem o passe livre, mas, no momento, necessita de transporte especial. Segundo a Secretaria de Transporte, ele não tem o perfil para solicitar este benefício. Em relação às medicações para hipertensão e diabetes, não as recebe pelo simples motivo de não ser cadastrado no programa de hiperdia, mesmo residindo em frente ao posto de saúde há dez anos. As atividades de lazer extramuros do CAPS estão suspensas por falta de transporte. Enfim, foram muitos os impasses e milhas de passos entre um serviço e outro, em busca de atender as necessidades do 'Senhor G', e que se somaram ao diagnóstico de alcoolismo, transtorno psiquiátrico - outros problemas clínicos tão importantes quanto os problemas iniciais. Buscamos contorná-los investindo no diálogo interprofissional, nos vínculos relacional e institucional, na escuta ativa, na negociação intersetorial. De modo geral, as alterações feitas no projeto terapêutico focaram em promover a inserção social do 'Senhor G' por meio de restabelecimento de vínculos familiar e institucional, que estavam fracos por conta da desorganização de vida.

\section{O experienciado: multifaces, desvendando conexões produzidas}

Percorremos juntas, aluna e preceptora, por determinado tempo, o caminho da formação profissional. Discordamos de algumas coisas, concordamos em outras, nos juntamos a outros sujeitos com distintos projetos de vida. Tivemos, nessa convivência, diferentes encontros, deixando um pouco de nós, levando algo do outro. Primordialmente, aprendemos. Por isso mesmo, podemos agora contar.

"A função de preceptor teve algumas particularidades, por ser uma assistente social orientada para o exercício profissional ético que prima pelos direitos dos usuários e também por atuar em um dispositivo da saúde como um Centro de Atenção Psicossocial (CAPS). Neste serviço, e a partir da posição de preceptora, me foi possível experenciar o que é a construção do vínculo institucional entre os profissionais, como também entre profissionais em formação, usuários e família, o exercício da escuta e da autonomia - recursos que se fazem necessários, independente da posição que ocupa o profissional ou sua especialidade". (Senhora P)

“Ao ingressar no PET-Saúde como aluna da graduação, não tinha ideia da proporção de recursos que um profissional em formação pode mobilizar e acessar de si para aprender a cuidar do outro, sem desprezar, mas, ao mesmo tempo, sem depender de recursos estritamente materiais ou tecnicistas. É comum para nós estudantes ansiarem por este aprendizado técnicoaplicado que pretende responder problemas na dureza dos fundamentos do conhecimento. Nem sempre é assim. Também não é tão simples amadurecer a esse nível de compreensão". (Senhora A)

Um modo de formação no PET é colocar o aluno na posição de observador participante, possibilitando vivenciar intensamente a função de mediador na construção do vínculo entre instituição e o usuário a partir de outro recurso também muito importante: a empatia, que deve se dar entre profissional e usuário. 
“No processo de elaboração do projeto terapêutico do 'Senhor G', nos foi possível vivenciar o que tem de mais rico no nosso trabalho. Uma construção de mão dupla, aluno e preceptor, em que o conjunto de saberes de forma horizontal nos levou a construção do projeto terapêutico, utilizando diversas ferramentas apontadas no campo da saúde como imprescindíveis para o exercício ético do cuidado". (Senhora P)

O Projeto Terapêutico Singular é um instrumento fundamental na materialização do cuidado e instrumentalização do profissional. Utilizado pela equipe do CAPS/ad, tem como direção de trabalho o cuidado por meio de diferentes estratégias preferencialmente coletivas.

“Acompanhando o 'Senhor G', pude perceber que é possível pautar o cuidado numa outra prática: da escuta ativa, na presença, da disponibilidade. Que é possível encontrar novos modos de nos conectarmos com usuário, família, serviços, menos prescritivos, deixando de lado os encaminhamentos frios nada resolutivos, que mais confundem do que direcionam. Que nos espaços institucionais ainda que com deficiências estruturais, neles além do óbvio de oferecer terapias curativas, operam como um lugar que agrega possibilidades de encontros e acontecimentos, capazes de resgatar autonomia dos sujeitos. De articular universidade e comunidade por intermédio da interação entre profissionais da academia, profissionais dos serviços, usuários e família". (Senhora A)

“Enquanto preceptora percebo que o maior desafio é provocar o aluno para que se exerça, mesmo sendo um profissional em formação, utilizando estas ferramentas e recursos. Isto acontece ora em maior ou menor medida, mas a forma como o trabalho se dá nos possibilita identificar os diversos recursos que o aluno tem e pode explorar. Este é um trabalho que considero artesanal e que nos exige uma escuta diferenciada dos limites e possibilidades de cada aluno". (Senhora P)

Como dissemos, o CAPS/ad é um serviço de base territorial, em que a clientela assistida traz, em seu pedido de ajuda, muitos outros pedidos; entre eles, muitas complicações clínicas. Na tentativa de darmos conta desses pedidos, buscamos construir um cuidado, e, para isto, temos, primeiramente, de desconstruir, junto ao aluno, a ideia de que há uma rede de assistência pronta. Isto porque o que chamamos de rede não está pronto e só acontece a partir das articulações permanentemente construídas para obtermos, como efeito, respostas às necessidades de cada usuário em tempos diferentes.

"Acompanhando o 'Senhor G', ouvi e vi muitos lamentos, queixas e sofrimento: morte de parentes queridos, separação conjugal, abandono dos familiares, maus tratos por profissional da saúde, morte violenta de usuário pós-alta. Mas também presenciamos abraços, alegrias, gratidão: o sobrinho que decidiu ajudar, a visita da irmã afastada, a festa de confraternização, a oficina de reforço escolar. Em todos esses momentos, precisei encontrar em mim atitude para lidar com cada situação, sobretudo contei com a presença da minha preceptora e dos colegas do grupo, cada um com olhar distinto, visto o perfil diferenciado de formação. Aprendi que conhecer e estabelecer vínculos, sejam, institucional, relacional, terapêutico, também é aprender a ser profissional". (Senhora A)

Criar vínculos implica permanências de relações tão próximas e tão claras que nos sensibilizamos com todo o sofrimento do outro, nos sentimos responsáveis pela vida e morte do paciente, possibilitando uma intervenção nem burocrática nem impessoal ${ }^{6}$. 


\section{Alguns efeitos das conexões produzidas no caminho e a produção de vínculo}

A experiência no PET desvelou alguns elementos considerados pelas narradoras como diferencial para formação do profissional de saúde e da importância de uma aprendizagem multiprofissional propiciada pelo PET-Saúde. Um dos principais desafios enfrentados pelo grupo PET foi manejar situações para viabilizar o processo terapêutico do usuário, diretamente ligado à forma de organização das redes de atenção à saúde. Percebeu-se também, por parte de alguns profissionais, pouca compreensão dos sentidos de integralidade do cuidado para além da estrutura física dos serviços.

Contudo, destacamos substanciais contribuições para a formação em serviço propiciada pelo PET, como: a oportunidade de aplicar novos conhecimentos, aprender a trabalhar em equipe, enriquecimento da aprendizagem por meio de mobilização de recursos internos, diversificação dos cenários de prática pautados na atuação na comunidade. As reuniões de equipes das quais participamos nos pareceram uma ferramenta importante de integração dos profissionais. Por mais que, algumas vezes, a pauta programada tenha cedido a discussões divergentes, essas fomentaram ações de educação permanente importantes para a problematização das questões do trabalho em saúde no SUS.

Quando pensamos na experiência como elemento da formação profissional, o PET-Saúde/UFF toma a experiência como um elemento central na formação, experiência como aquilo que nos acontece ${ }^{7}$. É preciso entender como e em quais situações os sujeitos da experiência são afetados; pode-se aprender acionando sentidos fisiológicos, um único sentido, ou associando vários deles ${ }^{8}$. Aprende-se com imagens, ouvindo, no toque na pele, no paladar, pelo cheiro. A formação pelo trabalho em saúde, no mundo do trabalho em saúde, convida o aluno a experimentar, pelo cuidado, encontros orgânicos com capacidade de produzir efeitos de problematização, que supõem a ação transformadora, inseparável do ato cognoscente, e, como ele, inseparável das situações concretas, mesmo que ocorra sobre os conteúdos já elaborados ${ }^{9}$. Nesse caso, pensa-se sobre o vivido e sobre si referenciando ao contexto, pois a problematização parte de situações vividas e implica um retorno crítico tendo os saberes previamente herdados incorporados à experimentação, como ponto de partida de construção do conhecimento ${ }^{6}$ :

O experimentar como ingrediente da formação nos desafia, sobremaneira, a construir estratégias pedagógicas que sejam capazes de deixar vazar as multiplicidades dos sujeitos em um coletivo múltiplo, com encontros precários. Precários na sua inconcretude e infinitude, na produção de subjetivação, experimentando no acontecimento. (p. 318)

Deste modo, vivenciamos diferentes momentos na produção do cuidado, que incide, diretamente, na formação do profissional, que, muitas vezes, se frustra diante da precarização das relações de trabalho, como, também, na precarização dos recursos e insumos básicos para uma assistência com qualidade.

O acolhimento e o vínculo foram estabelecidos como pilares fundamentais para a produção do trabalho em saúde. O acolhimento, quando realizado de maneira eficaz, aumenta a disponibilidade de ações e serviços a serem oferecidos aos usuários, contribuindo, assim, para maior satisfação dos mesmos, estimulando o vínculo com os profissionais e, consequentemente, a autonomia e cidadania dos usuários ${ }^{10}$.

Os espaços de supervisão com preceptor e as reuniões coletivas com todos os componentes do PET proporcionaram, à aluna, o encontro com noções e conceitos que, na academia, são difíceis de serem ensinados, talvez impossíveis de serem transmitidos e somente a experiência em ato possibilite, aos alunos, apreenderem esses conceitos centrais para o cuidado em saúde.

As apostas de cuidados são desenhadas no cotidiano dos serviços, de modo a construir, junto com usuários, ferramentas com potencialidades de ampliar a vida e projetos terapêuticos ${ }^{7}$. São arranjos construídos na produção de ações e planejamentos que convocam profissionais a compartilharem da vida do usuário, evidenciando ou não multiplicidades de existência. 


\section{Considerações finais}

Ampliar os cenários de atuação dos estudantes de graduação em saúde e fomentar a educação interprofissional tem sido uma das estratégias perseguidas pelos grupos do PET-Saúde/UFF, em Niterói. Essas estratégias abarcam inúmeros desafios e contribuições que vêm, sobremodo, reconfigurando o campo da formação e do cuidado em saúde. A partir da experiência do PET, os alunos desvendam um modo de cuidar que vai além da sala de aula, pois a produção do cuidado no serviço é atravessada por afetos, pela necessidade de disponibilidade para o inusitado, estabelecimento de vínculos, entre outros elementos. No entanto, tal constatação não seria possível numa proposta de formação tradicional, por isso, a importância do PET para interferir nos modos de fazer e ensinar das universidades. $O$ PET proporciona uma experiência marcante para a formação à medida que provoca os alunos a se exercitarem de outra maneira nos serviços de saúde.

Ao fim deste estudo, pode-se identificar a perspectiva de que a construção de vínculos mantém estreita relação com a construção diversificada dos arranjos que estão postos no sistema de saúde brasileiro. Arranjos que se estabelecem nos microprocessos dos serviços e que afetam, diretamente, a vida de usuários e profissionais, influenciando: a forma de organização da oferta e produção de serviços de saúde, controle de agendas, fluxo de acesso, diversidades de propostas terapêuticas, enfim, uma produção de cuidado autorizada diante de certa emergência requisitada tanto pelos usuários, quanto pelos profissionais que buscam acolher o sofrimento dos usuários.

Demandas trazidas por esses sujeitos forjam novas fórmulas, que se misturam legitimamente ao cotidiano dos serviços, ainda que esbarrem com crises de descrédito no SUS, no corporativismo de especialidades, na competitividade dos espaços de saber e fazer profissional. Um desafio a ser manejado pelos profissionais nos serviços de saúde e educação, para que novos modos de ensinar e aprender produzidos potencializem a vida de usuários, dos futuros profissionais e equipes dos serviços, para que estes assumam novas práticas de cuidar no SUS.

\section{Colaboradores}

As autoras trabalharam juntas em todas as etapas de produção do manuscrito. 


\section{Referências}

1. Berger Filho RL. Educação profissional no Brasil: novos rumos. Rev Iberoamer Educ. 1999; (20):87-105.

2. Ministério da Saúde. Portaria Interministerial $n^{\circ} 1.802$, de 26 de agosto de 2008. Institui o Programa de Educação pelo Trabalho para a Saúde - PET-Saúde. Diário Oficial União. 25 jun 2007.

3. Silva DGV, Trentini M. Narrativas como técnica de pesquisa em enfermagem. Rev Latinoam Enfermagem. 2002;10(3):423-32. http://dx.doi.org/10.1590/S010411692002000300017

4. Fonseca JA, Costa BR, Almeida CMOX, Silva GE, Senna MAA. PRO-PET Saúde na linha do cuidado da Rede Cegonha: integralidade e acesso numa unidade de saúde em Niterói/ RJ. Comunidade de Práticas. 2013 [acesso em 26 ago 2014]. Disponível em: http:// atencaobasica.org.br/relato/1415

5. Ministério da Saúde, Secretaria de Atenção à Saúde, Núcleo Técnico da Política Nacional de Humanização. Clínica ampliada, equipe de referência e projeto terapêutico singular. $2 a$ ed. Brasília, DF: Ministério da Saúde; 2008. (Série B. Textos básicos de saúde).

6. Abrahão AL, Merhy EE. Formação em saúde e micropolítica: sobre conceitosferramentas na prática de ensinar. Interface (Botucatu). 2014;18(49):312-24. http:// dx.doi.org/10.1590/1807-57622013.0166

7. Abrahão AL. Arranjos conceituais para a gestão em saúde a partir da Análise Institucional: relação entre gestão e subjetividade nas equipes da saúde. In: L'Abbate $S$, Mourão LC, Pezzato LM, organizadores. Análise Institucional e saúde coletiva no Brasil. São Paulo: Hucitec; 2013. p. 315-32.

8. Cunha MI. Aprendizagem ao longo da vida e avaliação do desempenho profissional. Avaliação (Campinas). 2011;16(3):559-72. http://dx.doi.org/10.1590/s141440772011000300004

9. Freire P. Educação como prática da liberdade. 29a ed. Rio de Janeiro: Paz e Terra; 1999.

10. Campos GWS. Considerações sobre a arte e a ciência da mudança: revolução das coisas e reforma das pessoas: o caso da saúde. In: Cecilio LCO, organizador. Inventando a mudança na saúde. 2a ed. São Paulo: Hucitec; 1997. p. 29-87. 
Este estudo apresenta as experiências de uma acadêmica de enfermagem e uma assistente social preceptora que atuam no Programa de Educação pelo Trabalho para Saúde. O objetivo consiste em ampliar o debate sobre a potência de provocar espaço de produção de vínculo no ambiente de trabalho, articulado à formação em saúde. A narrativa considera os profissionais em formação atuando no serviço e as formas de repercussão na formação em saúde. O relato recorta o acompanhamento de um usuário da rede de saúde mental pelos diversos pontos de cuidado, sendo o fio condutor da experiência. Foram empregados, como ferramentas, o método do aluno-sombra e o diário de campo. Considera-se que, a partir da experiência do PET, os alunos desvendam um modo de cuidar fora da sala de aula e próximo às reais necessidades do usuário.

Palavras-chave: Educação em saúde. Pessoal de saúde. Prática profissional.

\section{Health education and bonding: an experience with Education by Work for Health Program (PET-Health) in the health service network of Niterói, Rio de Janeiro State, Brazil}

This paper presents the experience of a nursing scholar and a social work preceptor who worked at the Education by Work for Health Program (PET-Health) with the goal of broadening the debate on the power of work environment bonding and its association with health education. This narrative included undergraduate professionals in service and the impact on health education. The report utilized the history of a person who used mental health services through various points of care as the common thread of experience. The student-shadow method and field diary were used as tools. The results from the program experience suggested that the students discovered a way to provide care outside the classroom and closer to the real needs of the users.

Keywords: Health education. Health personnel. Professional practice.

\section{Formación en salud y producción de enlace: una experiencia PET-Salud} en la red de Niterói, Rio de Janeiro, Brasil

Este estudio presenta las experiencias de una estudiante de enfermería y una asistente social tutora que trabajan en el Programa de Educación en el Trabajo para la Salud (PETSalud). El objetivo consiste en ampliar el debate sobre la potencia de generar espacio de producción de enlace en el lugar de trabajo, articulado a la formación en salud. La narrativa considera a los profesionales en formación actuando en el servicio y la forma de repercución en la formación en salud. El artículo aborda el seguimiento de un usuario de la red de salud mental por los diferentes puntos de atención, siendo el hilo conductor de la experiencia. Fueron empleados como herramientas el método de alumno- sombra y el diario de campo. Se considera que a partir de la experiencia de PET, los alumnos descubren una manera de cuidar fuera del aula y en torno a las necesidades reales del usuario.

Palabras clave: Educación en salud. Personal de salud. Práctica profesional. 
